\title{
VIA: Analyzing Device Interfaces of Protected Virtual Machines
}

\author{
Felicitas Hetzelt \\ TU Berlin \\ file@sect.tu-berlin.de
}

\author{
Martin Radev \\ Fraunhofer AISEC \\ martin.b.radev@gmail.com
}

\author{
Robert Buhren \\ TU Berlin \\ robert.buhren@sect.tu-berlin.de
}

\author{
Mathias Morbitzer \\ Fraunhofer AISEC \\ mathias.morbitzer@aisec.fraunhofer.de
}

\author{
Jean-Pierre Seifert \\ TU Berlin \\ jpseifert@sect.tu-berlin.de
}

\begin{abstract}
Both AMD and Intel have presented technologies for confidential computing in cloud environments. The proposed solutions - AMD SEV (-ES, -SNP) and Intel TDX - protect Virtual Machines (VMs) against attacks from higher privileged layers through memory encryption and integrity protection. This model of computation draws a new trust boundary between virtual devices and the VM, which in so far lacks thorough examination. In this paper, we therefore present an analysis of the virtual device interface and discuss several attack vectors against a protected VM. Further, we develop and evaluate VIA, an automated analysis tool to detect cases of improper sanitization of input recieved via the virtual device interface. VIA improves upon existing approaches for the automated analysis of device interfaces in the following aspects: (i) support for virtualization relevant buses, (ii) efficient Direct Memory Access (DMA) support and (iii) performance. VIA builds upon the Linux Kernel Library and clang's libfuzzer to fuzz the communication between the driver and the device via MMIO, PIO, and DMA. An evaluation of VIA shows that it performs 570 executions per second on average and improves performance compared to existing approaches by an average factor of 2706. Using VIA, we analyzed 22 drivers in Linux 5.10.0-rc6, thereby uncovering 50 bugs and initiating multiple patches to the virtual device driver interface of Linux. To prove our findings' criticality under the threat model of AMD SEV and Intel TDX, we showcase three exemplary attacks based on the bugs found. The attacks enable a malicious hypervisor to corrupt the memory and gain code execution in protected VMs with SEV-ES and are theoretically applicable to SEV-SNP and TDX.
\end{abstract}

\section{CCS CONCEPTS}

- Security and privacy $\rightarrow$ Virtualization and security.

\section{INTRODUCTION}

Cloud computing provides many advantages, such as on-demand resource allocation and high-availability guarantees. Nevertheless, many enterprises have not moved their data into the cloud due to security and privacy concerns [18]; one major concern being the compromise of the Hypervisor (HV) which has full access to the potentially sensitive data processed within the VM.

To protect data within cloud environments from a potentially malicious HV, several solutions have been proposed by academia [36, $58,62,64]$. In 2016, AMD proposed the first commercially available solution called Secure Encrypted Virtualization (SEV) [37], followed later by similar solutions from Intel [32] and IBM [31]. These solutions enhance the security of VMs, as the VM no longer depends on the HV's security or on the integrity of the cloud provider. While recent research has uncovered security issues specific to the design of the SEV technologies [21, 22, 25, 30, 42, 43, 46, 47, 60, 61], little research has been done in analyzing the VM's software under this new threat model [52]. Specifically, only limited effort has been spent on analyzing security risks due to drivers which communicate with devices controlled by the now untrusted HV. Drivers inside the VM should now treat input from virtual devices as malicious and should implement proper input validation. Compared to attacks from external hardware devices against the host OS $[45,48]$, validation is even more critical in a protected VM as a malicious HV has fine-grained control over the VM, greatly enhancing the adversary's abilities. For example, a HV is able to intercept the VM's execution at an arbitrary point in time and can monitor various feedback channels to infer the execution state of the VM [43].

Identification of drivers performing insufficient validation of $\mathrm{HV}$ controlled input requires the analysis of a vast codebase. To be able to quickly identify missing input validation in such a vast code base, we present VIA. VIA is a fuzzing framework targeted to detect missing input validation in device drivers of VMs. Although dynamic analysis tools exist for the device interface, they require specialized hardware [45] or devices implementations [55] and target only a limited subset of driver functionality. Existing device independent approaches target the USB interface $[27,50]$ and a limited subset of the Peripheral Component Interconnect (PCI) [56] interface. Notably, none of the device independent approaches targets the complex DMA interface of device drivers, which has been shown to be a rich source of vulnerabilities in the past [45]. VIA supports Virtual I/O (VirtIO), Platform and PCI device interfaces -including DMA- allowing VIA to target a large extent of the virtual device driver interface.

In addition to limited bus support, previous approaches require a full VM setup and suffer from poor performance due to delays embedded in device drivers, frequent context switches between the host and the VM and inefficient interrupt injection. To address these issues, VIA moves the fuzzing setup and the Linux kernel into a userspace program and thereby eliminates the need for a full VM setup including userspace and virtualization software. VIA further increases analysis throughput by applying optimizations to the Linux kernel environment that reduce redundant delays in driver code. To inject fuzzing data into the virtual device interface, VIA employs in-process IO-interception and avoids costly VMEXIT- or pagefault-based approaches. Further, VIA incorporates information about the target driver state to efficiently guide the analysis through targeted interrupt injection. 
Using VIA, we analyzed 22 device drivers. We identified 50 cases of missing input validation and initiated multiple patches to the virtual device driver interface [3-12]. In total, we found issues in 20 of the 22 analyzed drivers. To underline the severity of the missing validations, we detail three cases of how a malicious $\mathrm{HV}$ is able to execute arbitrary code or corrupt protected memory within a VM. To evaluate VIA, we compare our approach against Agamotto [56], a state-of-the-art, VM-based, PCI device fuzzer. Our evaluation shows that, VIA on average improves the analysis throughput by factor of 2706 and the percentage of driver code covered by a factor of 2.26 .

In summary, our contributions are:

- Analysis of the virtual device attack vector. We identify and describe the virtual device interface as a new attack vector against protected VMs. This new attack vector applies to all technologies that aim to isolate complete commodity operating systems.

- Categorization of bug classes. We identify and describe numerous bugs in the virtual device driver interface, which we classify by type and severity. To demonstrate the severity of the identified bugs, we describe three proof of concept exploits to obtain code execution and corrupt memory in a protected VM.

- Targeted dynamic analysis tool. We design, implement and evaluate VIA, a dynamic analysis tool designed to detect missing sanitization of HV-controlled data. VIA comprises of a userspace fuzzer with improved bus support and performance. We detail three optimizations that were applied to improve dynamic analysis: in-process IO interception, delay reduction and targeted interrupt injection.

\section{BACKGROUND}

The industry has proposed different security technologies that aim to protect VMs in an untrusted environment. This work focuses on technologies for the $\mathrm{x} 86$ architecture: AMD's SEV and Intel's Trust Domain Extensions (TDX). This section provides background on both technologies and discusses the use of devices in virtualized environments.

\subsection{Protected Virtual Machines}

AMD SEV is the first commercially available technology aimed at protecting VMs from a potentially malicious HV [37]. Protection is achieved by encrypting the VM's memory with a secret key, that is unique to the VM and inaccessible by the cloud provider. The memory encryption, key management and VM-entry and -exit are handled by a separate highly privileged component: the Platform Security Processor (PSP). Each guest controls the encryption of its memory via a dedicated bit in its private page table. Unencrypted, also named shared, pages can be used to exchange data with the HV. Later, AMD released SEV-ES which enhances VM protection by encrypting and integrity-protecting the VM's registers. Another improvement - SEV-SNP - was announced in 2020 which protects the emulation of special instructions, as well as the nested page tables against manipulation. Intel recently proposed a similar technology to SEV-SNP, called TDX [32]. Notably, all these technologies improve security for the protected VM through architectural changes, but do not directly address vulnerabilities in the VM's software.

At the time of writing, only SEV and SEV-ES are officially supported in Linux and by available CPUs. AMD SEV-SNP and Intel TDX are not officially supported on currently available CPUs, but patches to open-source projects have been made public for both $[17,34]$.

\subsection{Device Virtualization}

While the hardware virtualization extensions provide CPU and memory virtualization, device virtualization is performed by the $\mathrm{HV}$ and requires cooperation from the VM. The VM discovers attached devices by traversing known bus addresses and then reading the identifiers and configuration advertised by the HV. The VM then loads and initializes the corresponding device driver. The driver proceeds with programming the device through either regular access to Memory-mapped I/O (MMIO) or special Programmable I/O (PIO) instructions.

While MMIO and PIO are used to send commands and receive configuration information from the device, the communication of large messages, like network packets, happens through DMA. A driver is responsible for allocating physical memory buffers and providing their physical addresses to the device. When the device decides to provide a payload to the VM, the device copies the data to the DMA buffer and notifies the driver that new data is available. The driver can then read the data and process it.

The transparent use of virtual devices with TDX or SEV requires changes in guest operating systems such as Linux to accommodate for the fact that the HV cannot read a guest VM's private memory. At the kernel initialization stage, the VM's Linux kernel makes adjustments to the DMA and MMIO API implementation to use decrypted memory for communication. The device and the corresponding driver can use the memory for sharing frequently accessed communication structures like buffer descriptors and ring queues without the need for memory synchronization. Next, the swiotlb component is enabled, which allocates and manages a large contiguous decrypted memory area. When a driver uses the streaming DMA API to map a buffer without coherency requirements (e.g., dma_map_single), the implementation internally returns the physical address of a buffer in the swiotlb decrypted memory region. When the driver synchronizes the memory using the DMA API (e.g., dma_unmap_single), the swiotlb implementation copies (bounces) the memory from the decrypted memory region to the corresponding private buffer of the driver.

\section{THREAT MODEL}

Our threat model assumes a malicious actor who controls the HV and seeks to compromise a protected VM. The malicious actor may have gained access to the HV by exploiting a system vulnerability or may be a rogue server administrator. The HV cannot directly read or modify the VM's state, as the VM's memory and register contents are encrypted. The VM runs an up-to-date operating system with a Linux kernel.

The HV manages access to hardware devices for the VM by providing the appropriate virtualized device in the cloud setting. 
The virtual device can be based on VirtIO or on a custom interface, but the VM's Linux kernel must support the device. The HV can configure arbitrary devices during the VM's boot and attach or detach any hot-pluggable device during the lifetime of the VM.

Being in control of the virtual device, the malicious HV is able to modify any data structure shared with the device. For example, such structures include descriptor buffers and ring queues commonly used in many devices. The HV can intercept MMIO and PIO operations and provide any value for MMIO reads and the IN instruction. Additionally, the HV is able to intercept and modify any communication between the real hardware device and the VM's driver. For example, the HV can modify the contents of network packets sent to the VM via the DMA interface. Further, the HV is able to inject interrupts into the VM, as necessary for sending a notification from a virtual device to the VM's kernel.

This proposed threat model precisely captures the threat models of AMD SEV Secure Nested Paging (SEV-SNP) [15] and Intel TDX [33]. Any attacks on the proposed threat model are also applicable to both of these technologies.

\section{DESIGN}

In this Section we detail the high level design of VIA. First, we give an overview of the architecture of VIA as well as VIA's central components. Further, we present considerations about how the design avoids false positives during bug discovery and describe options to extend VIA.

VIA is the first comprehensive approach to detect missing sanitization of data provided by a virtual device to a Linux device driver. It extends the scope of current hardware interface analysis by targeting VirtIO, PCI, and Platform device interfaces. In addition to opening up these new interfaces for analysis, VIA also addresses performance issues in existing approaches.

The dynamic analysis framework comprises of a feedback-driven, in-process, userspace fuzzer that mutates data sent from a virtual device to the device driver.

On a high level, VIA provides device simulations of varying complexity that forward random data to the HV interface of device drivers. The drivers are loaded as shared libraries into a userspace program that provides a Linux kernel environment. VIA extends and modifies the Linux kernel environment to support the analysis. Namely, VIA applies delay reduction, targeted interrupt injection and in-process IO-interception to improve fuzzing throughput significantly. Further, VIA extends the fuzzer's input generation mechanism to support the analysis and avoid deadlocks in the driver code.

System Overview. Figure 1 illustrates the overall design of VIA's dynamic analysis framework. The two main components are the Fuzzer-Engine and the Library-OS. The Fuzzer-Engine is responsible for setup, monitoring, and controlling of the test execution. It processes code coverage feedback and provides mutated data that the analyzed Driver consumes. Additionally, it contains an exchangeable Harness that drives interaction between the simulated Device and the driver. The Library-OS component contains the Linux kernel code within which we execute the driver, and software implementations for PCI, VirtIO, and Platform devices that provide mutated data to the driver.

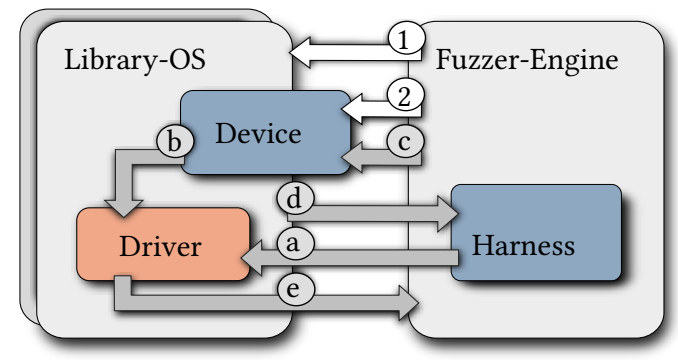

Figure 1: High-level overview of VIA. White arrows indicate initialization, gray arrows indicate actions performed during each fuzzing iteration. The main components are the Fuzzer-Engine and the Library-OS, which again contain the Harness and the analyzed Driver that is bound to the according virtual Device.

In order to start the analysis, the Fuzzer-Engine first starts one or more instances of the Library-OS (1) and configures the software device of each instance according to the selected device type (2).

During each fuzzing iteration, the Fuzzer-Engine executes the instructions provided by the harness. The harness interacts with the Library-OS via the standard Linux system call interface (a), which we extended to facilitate interrupt generation and device driver (un-)initialization. The role of the harness is to issue a set of system calls to (i) initialize the driver, (ii) trigger interactions between the driver and the device (b) and (iii) un-initialize the driver. When the driver requests data from the device, the device will either forward mutated data from the Fuzzer-Engine (c) or handle the request according to its internal device simulation logic. In order to resolve locks quickly, the Library-OS notifies the harness if a thread initiated by the driver is waiting on a resource (d); the harness then has the option to trigger an interrupt in order to resolve the block. After each execution of the harness, the code coverage generated by the driver is forwarded to the Fuzzer-Engine (e) to guide future mutations.

Device Simulation. VIA simulates virtual devices in order to communicate with the HV interface of device drivers. The FuzzerEngine configures the device according to a configuration file provided by the analyst. Depending on the device type (PCI, VirtIO, or Platform), VIA uses different approaches to simulate the device. The device simulation generally falls into one of two categories: passthrough and emulation.

Both passthrough and emulated devices perform an initial device specialization based on a configuration file. The initial specialization configures device identifiers that allow matching the driver to the device. In the case of Platform devices, drivers are matched via their name [13]; PCI and VirtIO devices are matched via Vendor- and Device-IDs [14, 29]. Additionally, VIA configures the device's resources at this stage, namely interrupts and MMIO and PIO regions that the driver expects to be provided by the device.

VIA uses passthrough for PCI and Platform devices. After the initial configuration, the simulated device only forwards mutated data to the driver and ignores data sent from the driver to the device. Interrupts have to be triggered by the harness. 
The emulation mode allows VIA to target higher layers of the driver code. To that end, emulated devices adhere to the interface specification (e.g., [14]) to establish basic communication channels between driver and device. Therefore, emulated devices process data sent from the driver to the device and automatically trigger interrupts depending on the device's internal state. VIA can simulate VirtIO devices in both passthrough and emulation mode. While passthrough simulation targets the lower levels of the VirtIO driver stack, e.g., virtio-ring or virtio-mmio, emulation can reach code in higher layers, including drivers such as virtio-net and virtio-blk. VIA provides a single unified emulated VirtIO device stub to analyze arbitrary VirtIO drivers.

Targeted Interrupt Injection. Most driver-device interaction is initiated via the execution of interrupt handlers that are part of the driver. The harness therefore injects interrupts to trigger the interaction between the simulated device and the driver. However, interrupts should not be injected randomly as this might decrease performance as well as coverage stability. VIA addresses this issue by providing callbacks to inject interrupts precisely when an execution thread of the driver enters a blocked state while waiting on data from the device.

Delay Reduction. Driver execution is often prolonged during initialization or peripheral input processing. This is mostly due to properties of the real hardware device that the driver assumes to interact with. Through manual analysis of driver code, we found these delays to be mostly redundant for software-based device implementations. Therefore, VIA modifies common kernel interface functions to eliminate delays in a generic way without impacting the functionality of the driver. Note that during such delays the processor is not necessarily idle, as the Linux kernel might schedule other workloads intermediately. Therefore, approaches such as presented in ReVirt [26], that skip cpu idle time, are not directly applicable.

Intercepting IO. In order to inject data into the hardware interface, we have to intercept the normal communication path between driver and device. MMIO and PIO regions are accessed via a defined kernel interface. VIA extends the functions comprising this interface to inject test data during a fuzzing iteration.

For DMA, we have to distinguish between coherent and streaming DMA. Streaming DMA is synced once before and after device interaction; therefore, the whole DMA buffer is filled by the fuzzer at CPU synchronization points. Coherent DMA can be accessed continuously without explicit synchronization from the guest CPU. Therefore, fresh values should be provided at each access. However, the kernel does not offer an interface to hook such accesses. VIA instead facilitates the address sanitizer [44] instrumentation to intercept accesses to coherent DMA memory. Address sanitization adds instrumentation around memory accesses that manages values stored in a shadow memory map. Upon memory access the values in the shadow memory are modified to indicate the allocation state of the accessed memory area. Further each access is validated by examining the allocation state based on the values stored in the shadow memory. VIA marks the shadow memory corresponding to coherent DMA memory areas with a special value, which is processed by the address sanitizer error handling code. When handling read operations, the sanitizer's error handling code is augmented to inject the next value from the input stream at the faulting DMA address and then continue execution.

To improve performance, some memory access types are excluded from address sanitization. These include accesses to allocations on the current stack frame that are determined to be in bounds or promotable to register accesses and accesses to certain types of global variables. We found that, for the analyzed drivers, none of the consistent DMA allocations met these criteria.

Kernel addresses exposed to the HV can leak information about the address space layout of the VM. To detect such issues, all values exposed to the virtual device via MMIO, PIO or DMA are checked. If a value contains a kernel address a warning is issued.

Input Generation. Each fuzzer-generated input is treated as a serialized sequence of memory transfers from the device to the driver. Each time the driver requests data from the device, VIA consumes the corresponding number of bytes from the test input. If the generated input is exhausted, the input is extended with pseudo-random data. This ensures that the driver receives different values to avoid infinite loops and deadlocks.

Avoiding false positives. The adaptions of the Linux kernel environment were carefully designed to avoid false positives during bug discovery. To that end, the framework mimics the functionality of the swiotlb implementation in Linux, which is always enabled with SEV and TDX. Like the Linux swiotlb implementation, VIA keeps track of mapped DMA memory areas and checks if these areas are currently mapped before the unmap operation can be performed. If a DMA address has not been mapped previously the unmap invocation is rejected. Without such validation, drivers that pass device controlled DMA addresses to dma_direct_sync_single_for_cpu or dma_unmap_single, such as virtio-ring, sungem and sunhme, could be exploited to corrupt the VM's memory. Note that VIA uncovered bugs in the swiotlb implementation, as detailed in Section 6.2. To aid the analysis, VIA handles such issues and reports them.

While delay reduction could potentially lead to the detection of bugs that are not exploitable if the delays are re-introduced, the HV has fine grained control over the VM's execution and timers [52]. Therefore the HV can adjust scheduling and delays within the VM in order to aid the exploitation of such bugs. Since the HV controls interrupt generation under SEV as well as TDX, no false positives can be generated due to targeted interrupt injection.

Extending VIA. VIA's modular design allows for the automated analysis of a wide range of different device driver types. While this work focuses on PCI, VirtIO, and Platform devices, VIA is designed to be easily extensible to support other device types.

Table 1 presents the lines of code required to implement harnesses and device simulations. Depending on the internal complexity of the simulated device, the implementation can be very minimal as for PCI and Platform devices, or require higher implementation effort as for VirtIO. To add a new device driver of an already supported device type, the respective driver has to be enabled in the Linux kernel configuration and a configuration file containing the device identifiers has to be created. VIA already provides a default 
Table 1: Implementation complexity of harnesses and device simulations provided by VIA. All code is written in C. The number of lines of code is obtained using the tool cloc [16]

\begin{tabular}{lrrrr}
\cline { 1 - 1 } \cline { 4 - 5 } Device Type & LoC & & Harness & LoC \\
\cline { 1 - 3 } PCI & 242 & & Default & 20 \\
Platform & 79 & & Network & 73 \\
VirtIO & 554 & & Block/Char & 72 \\
\hline
\end{tabular}

harness implementation that performs one initialization and uninitialization of the driver in each fuzzing iteration. This default harness performs a basic analysis of arbitrary device drivers. Additionally, VIA provides more elaborate harness implementations for network, block, and character drivers, that interact with resources created by the driver and cover a larger extent of the driver's code base.

\section{IMPLEMENTATION}

In this section, we present the implementation details for VIA. Further, we detail the adaptions to the Linux kernel environment that enable the functionality described in Section 4.

VIA is based upon Linux Kernel Library (LKL) [51] and clang's libfuzzer [2]. The basic fuzzing infrastructure is provided by libfuzzer, which handles mutation, test-case scheduling, as well as test-execution and feedback. LKL provides the Linux kernel execution environment to load and interact with device driver code.

Instrumentation. To collect coverage feedback, we instrument the driver code with the recommended clang sanitizer to be used with libfuzzer: - fsanitize=fuzzer-no-link. To avoid feedback pollution, we only enable feedback collection on the target driver, omitting the remaining kernel code. To also enable address sanitization instrumentation on the whole kernel, we use the clang flags $-f$ sanitize=address - fsanitize-recover=address. The address sanitization provided by clang requires the $\mathrm{C}$ standard library (libc) to be used for heap management. Since LKL uses the Linux kernel allocation algorithms instead of the one provided by libc, we adapted the kernel address sanitizer to directly modify the shadow memory maintained by clang's userspace address sanitizer instead of maintaining its own separate shadow memory region.

Delay Reduction. In order to avoid unnecessary delays while fuzzing, the kernel's *delay, *sleep, schedule_timeout [_*], and time_before/after functions are modified when they are invoked from driver code. In the case of schedule_timeout, *delay, and *sleep the framework returns immediately.

In the case of time_before and time_after, the framework always returns that the queried time is after the specified time stamp. Timeouts, used in wait_event_* functions, are not disabled because they are necessary for the functionality of the driver. Additionally, the delay in queue_delayed_work is always zeroed-out.

Lock Tracking. To track blocked threads waiting for events, the functions wait_for_completion_* and *_wait_event_* are modified. Specifically, the functions are extended to store and remove
Table 2: List of examined drivers with their type, communication interface and number of discovered bugs. $Q V D$ stands for QEMU Virtual Device, and CVM for Google Confidential VM. We distinguish between low and high severity bugs. An analysis of each bug can be found in our repository [1].

\begin{tabular}{lccccc}
\hline \multirow{2}{*}{ Driver } & \multirow{2}{*}{ Usage } & Type & Bus & \multicolumn{2}{c}{ \#Bugs } \\
& & & & Low & High \\
\hline virtio_ring & QVD & Common & VIO & 2 & 4 \\
virtio_net & QVD & NET & VIO & 1 & 1 \\
virtio_blk & QVD & BLK & VIO & 5 & 1 \\
virtio_crypto & QVD & CRYPTO & VIO & 2 & 0 \\
virtio_rng & QVD & RNG & VIO & 0 & 1 \\
virtio_console & QVD & MISC & VIO & 1 & 0 \\
virtio_balloon & QVD & MISC & VIO & 2 & 1 \\
virtio_input & QVD & MISC & VIO & 0 & 0 \\
rocker & QVD & NET & PCI & 1 & 3 \\
sungem & QVD & NET & PCI & 1 & $0{ }^{*}$ \\
sunhme & QVD & NET & PCI & 2 & 0 \\
8139cp & QVD & NET & PCI & 1 & 0 \\
vmxnet3 & QVD & NET & PCI & 0 & 5 \\
ne2k-pci & QVD & NET & PCI & 0 & 0 \\
e100 & QVD & NET & PCI & 0 & 1 \\
e1000 & QVD & NET & PCI & 0 & 3 \\
e1000e & QVD & NET & PCI & 1 & 0 \\
qemu_fw_cfg & QVD & MISC & PLT & 1 & 0 \\
acpi & QVD & MISC & PLT & 0 & 1 \\
gve & CVM & NET & PCI & 2 & 3 \\
nvme & CVM & BLK & PCI & 1 & 1 \\
tpm_tis & CVM & TPM & PLT & 0 & 2 \\
\hline Device-controlled values passed to swiot1b_tbl_unmap_single as described in Sec- \\
tion 6.2 were not counted as separate bugs. & & & & \\
& & & & & \\
& & & & \\
& & &
\end{tabular}

handles to the blocked thread before and after completion of the event. Whenever a new entry is added, the harness is notified.

\section{EVALUATION}

In this section, we first categorize the bugs uncovered in the virtual device interface and present three exploits based on the discovered bugs. Finally, we evaluate the performance of VIA.

\subsection{Bug Overview}

VIA was used to examine a total of 22 drivers in the Linux kernel with version 5.10.0-rc6. Of the analyzed drivers, 19 belong to QEMU virtual devices and 3 to specialized hardware available in Google's SEV-enabled confidential computing VM [28]. The analysis was performed via intermittent runs over the course of several months (i.e., from November 2020 to February 2021). Each fuzzing session usually lasted between a few minutes to several hours. We responsibly disclosed found security issues with the respective maintainers. To prepare the drivers for the dynamic analysis, we patched problematic code where possible by adding proper sanitization. Additionally, we removed complex checks (e.g., checksum comparisons) on data provided by the fuzzer. This optimization was applied to the drivers e1000, rocker and sungem. Addressing such 
Table 3: Distribution of bug classes found by VIA.

\begin{tabular}{lc}
\hline Bug Class & Count \\
\hline Out-of-Bounds access & 14 \\
Invalid memory access & 10 \\
Slab management & 8 \\
Device-shared pointer & 5 \\
Miscellaneous & 3 \\
Assertion failure (BUG) & 4 \\
Unbounded allocation & 5 \\
Deadlock & 1 \\
\hline
\end{tabular}

issues in a generic way is an orthogonal problem that has been addressed in other works [19, 23]. The required modifications were easy to identify from the kernel or crash log, and workarounds could easily be developed. We list the changes to the driver code in our public repository [1].

In total, VIA uncovered 50 novel bugs across the drivers listed in Table 2. At the time of writing, 9 of the discovered bugs in swiotlb, gve, virtio_ring, virtio_net and vmxnet3 have been, or are currently being fixed [3-12]. The applied patches include simple corrections of copy paste errors [10] or return codes [9], additions of NULL pointer checks [3] and corrections of driver state updates $[4,11]$. More elaborate patches were applied to facilitate the exlcusion of control data such as pointers, indices or variables determining access length from device control $[5-8,12]$.

Each entry in Table 2 was carefully validated by examining the source code of the offending driver, and bug duplicates were removed. A thorough analysis of each bug is available in our repository [1]. The types of bugs covered include out-of-bounds access, device-shared pointer, invalid memory access, slab management bugs, miscellaneous bugs, assertion failure, unbound memory allocations, and deadlocks. We distinguish between bugs of high and low severity. We consider bugs as severe that potentially leak or corrupt guest memory either on their own or by aiding exploitation of different vulnerabilities. These include out-of-bounds access, device-shared pointer and slab management bugs, such as useafter-free or double-free. The remaining bug classes are considered low-severity, as they will impact the guest system's availability but likely do not undermine the security guarantees of SEV or TDX. Low-severity bugs usually arise from implementation errors, that should be fixed to guarantee proper functionality of the driver.

Table 3 lists the bug classes and the corresponding number of discovered bugs. Among the most common bugs are Out-of-Bounds access and Invalid memory access bugs, with respectively 14 and 10 incidents. The discovered root cause of these two classes is missing validation for the index and length fields of shared data structures. Next are slab management bugs encompassing 8 instances of use-after-free or double free issues. Such bugs often occur in the teardown code of a function if error handling is incorrectly implemented. Further, VIA uncovered 5 device-shared pointer bugs where a VM kernel pointer is stored in shared memory. These deviceshared pointer issues were all present in few network drivers (GVE, e100, rocker, vmxnet3). The miscellaneous bug category comprises of 3 unique bugs including missing synchronization of device memory, division by zero and limited directory traversal. Lastly, our fuzzing setup caught 4 cases of assertions failures, 5 unbounded memory allocations and 1 deadlock. The assertion failure class includes cases when the kernel would detect an unexpected state and call BUG(). The unbound memory allocations category considers cases for which the kernel performs large allocations or fails to free allocated memory, leading to out-of-memory conditions. Deadlocks can occur, if data from the device introduces a VM state in which a kernel thread is indefinitely waiting for a resource.

\subsection{A Case Study of Three Bugs}

In this section we discuss how a malicious administrator, with only access to the QEMU process, can exploit three of the discovered bugs to overwrite secret memory of a virtual machine and to gain code execution inside the virtual machine.

The exploitation of the three bugs was performed on a virtual machine protected with SEV Encrypted State (SEV-ES) and running Linux 5.10.8. The exploits were designed with Kernel Address Space Layout Randomization (KASLR) disabled, though a malicious HV can use leaked VM kernel pointers to infer the memory layout of the VM if required.

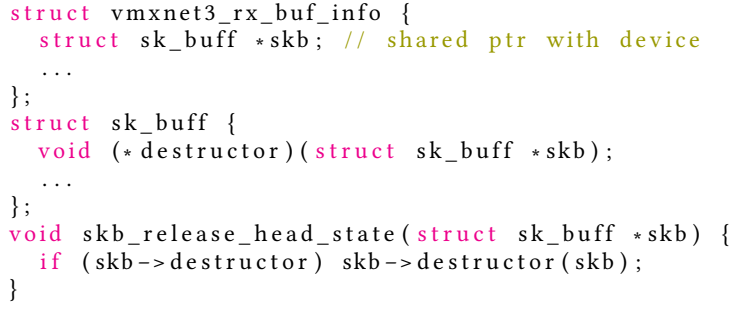

Figure 2: Vulnerable code in the vmxnet 3 driver. The pointer skb on Line 2 is accessible by the device and can be overwritten. By creating a fake skb object and overwriting the pointer, the device can trick the driver into calling any function on Line 12.

Device-shared pointer in vmxnet 3 . The vmxnet 3 interface relies on an array of descriptors that can be accessed by the device. A vmxnet 3 descriptor, shown in Figure 2, contains an associated skb kernel pointer among other fields. The pointer points to a preallocated sk_buff object in the VM's private memory, which can be used immediately when a network packet is received.

The exposure of this pointer to an untrusted device leaks information about the VM kernel's randomized address space and additionally allows a malicious HV to overwrite the pointer with a convenient value. When the packet is received, the contents of the sk_buff are parsed, and the object is eventually freed. Before freeing the packet, the sk_buff's destructor would be called, as shown on Line 12.

In order to exploit the bug the HV overwrites the skb pointer in the descriptor to point to decrypted memory at the moment of sending a packet to the VM. At the same time, the HV forges a fake sk_buff object at the new address and overwrites the destructor 
pointer to a suitable code gadget in the VM's kernel. The code gadget would position the stack into decrypted memory, which would then allow continuing execution using return-oriented programming. We determined empirically that many code gadgets are suitable destructor pointers because many registers and the stack contain the address of the skb in decrypted memory. Similar exploitation opportunities were found in the driver implementations of GVE, e100, and Rocker.

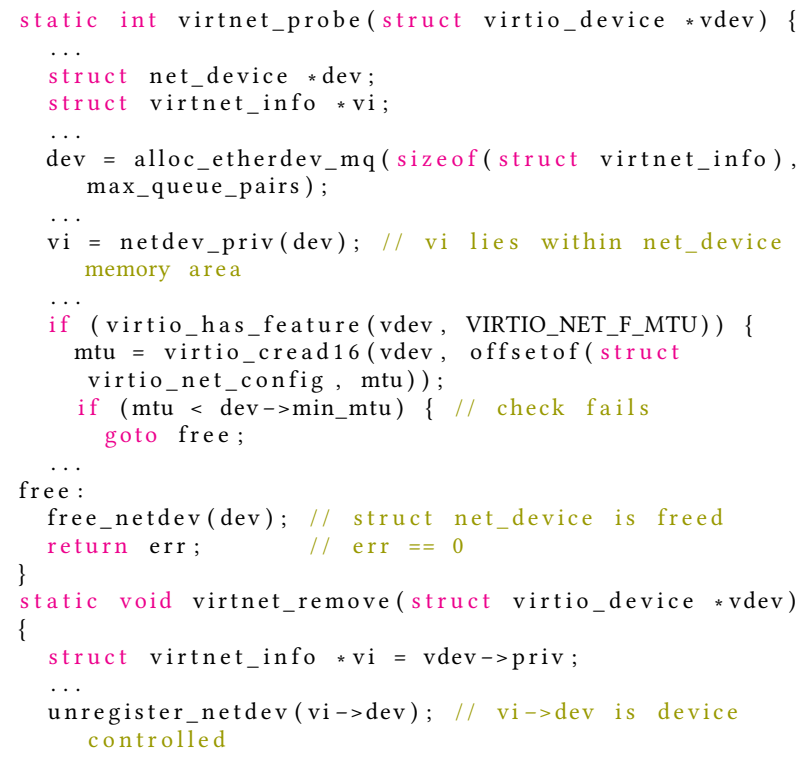

Figure 3: The HV can induce virtnet_probe to fail by providing a low mtu value. The driver fails to set err correctly. This leads to access of the freed memory, pointed to by vi, during device removal.

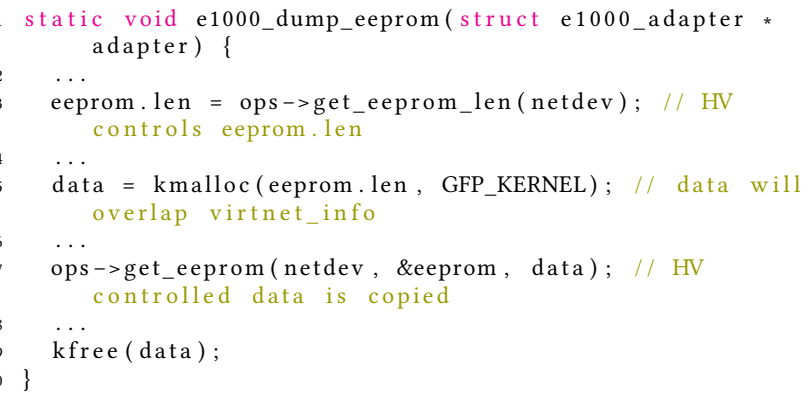

Figure 4: The function e1000_dump_eeprom is used to gain control over the memory freed in virtnet_probe. The HV controls eeprom. len. The function get_eeprom fills the allocated buffer with data controlled by the HV.

Use-after-Free bug in VirtIO-Net. The virtio_net driver fails to return an error code if the initialization fails due to an invalid $\mathrm{mtu}$ value provided by the HV. This leads to a use-after-free bug when the device is removed. The vulnerable code is depicted in Figure 3. To exploit this bug the HV first attaches the virtio_net device and causes virtnet_probe to fail by providing a low mtu value. To obtain control over the freed memory area, the HV then attaches the e1000 device and triggers the code path shown in Figure 4. Specifying an eeprom. len of $0 \times 1000$ will cause a new allocation in the kmalloc- $4 k$ cache, which overlaps the memory area of the freed struct virtnet_info. The structure virtnet_info contains the field dev, which points to a net_device structure. The net_device structure contains the function pointer ndo_uninit, that will be called in unregister_netdev via the invocation of virtnet_remove. Similar to the previous exploit, the HV overwrites virtnet_info->dev to point to a decrypted memory area and sets ndo_uninit to point to a suitable code gadget. Next the HV detaches the virtio_net device to trigger the vulnerable code path and gain code execution.

The reliability of the exploit relies on two factors. First, the allocation of the data allocated in e1000_dump_eeprom has to overlap the freed struct net_device. Second, the re-allocated memory area should not be overwritten before virtnet_remove is called. Under SEV-(-ES, -SNP) the HV has read access to encrypted memory. This allows the HV to infer the host virtual addresses of both allocations by monitoring changes to encrypted memory at suitable points during the interaction between the device and the driver ${ }^{1}$. In order to verify whether the data was overwritten, the HV compares the encrypted memory state after e1000_dump_eeprom to the current state before detaching the virtio_net device. If the inferred addresses differ or the memory has been overwritten the $\mathrm{HV}$ can either repeat the exploit attempt by attaching a new device, or choose to abort the attempt.

Out-of-Bounds bug in VirtIO / swiotlb. Every VirtIO driver in Linux relies on a common VirtIO interface implementation that handles the state of each communication queue. Each queue is associated with an array of descriptors, depicted in Figure 5. When a device sends a payload to the driver, the device typically only reads but does not update the addr and len fields since they are provided by the driver.

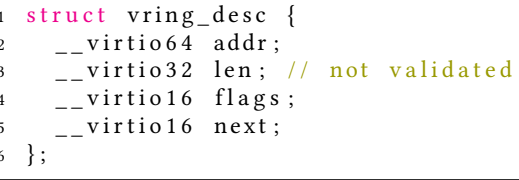

Figure 5: Contents of a VirtIO descriptor. The devicecontrolled value of len is not validated and is used as an argument to memcpy.

When a VM uses AMD SEV or Intel TDX, the VirtIO implementation relies on the DMA API and its swiotlb implementation. The swiotlb layer allocates a shared memory region used for communication. A device copies a payload to this region, the swiotlb layer performs some validations and finally bounces the payload to the

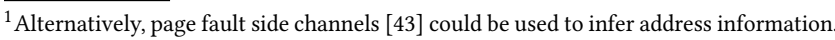


driver's private buffer when memory is synchronized. The length of the copied data is given by the len field of the vring_desc structure, shown in Figure 5.

Our fuzzing tool uncovered that the swiotlb layer does not validate the device-controlled length of the memory copy, which causes a buffer overflow. This allows a malicious device to overwrite the private memory of a protected VM with arbitrary data of arbitrary length. This vulnerability occurs in shared code by all Linux VirtIO drivers. What area of private memory can be overwritten depends on the memory allocator used by the driver. For example, virtio_console and virtio_rng would allow overflowing a buffer allocated from the Linux SLAB allocator. We verified empirically that net_device structures, e.g. allocated during the initialization of the e1000 device, are often allocated in proximity to the descriptor buffers allocated in virtio_console. In such cases the HV can exploit the out-of-bounds bug to overwrite the contents of the structure and craft an exploit similar to the previous example.

To increase the reliability of the exploit, the HV infers the host virtual addresses of the descriptor buffers as well as the net_device structures by observing changes in encrypted memory. Based on the inferred addresses, the HV selects a descriptor that points to a buffer that is allocated in proximity to one of the net_device structures and adjusts the descriptors len field to overwrite the net_device memory area. If no suitable locations are found the HV re-attaches the devices until a suitable combination of descriptor buffer and net_device addresses is detected.

\subsection{Performance Analysis}

In this section, we evaluate the performance of VIA. We compare VIA against a state-of-the-art VM-based approach. Further, we examine whether delay reduction and targeted interrupt injection are effective in improving fuzzing performance. We conducted all of our experiments on a machine equipped with an 8-Core Intel i7-7700 and 64GB of memory. The analysis of each driver was restricted to one CPU core, to eliminate differences in performance due to the multithreaded design of VIA. In order to reduce randomness, we used an empty initial corpus and a fixed random seed for all evaluations. The size of individual corpus files was limited to $10 \mathrm{kB}$ and a timeout of 120 seconds per fuzzing iteration was used. The harness modules used for the evaluation are listed in our public repository [1].

To evaluate the performance improvement compared to previous VM-based approaches, we compare VIA against Agamotto [56], which is a state-of-the-art PCI device fuzzer. Agamotto is similar to USBFuzz [50] in function and performance. It utilizes a virtual device which generates a mutated IO-stream that is forwarded to the target driver. In addition, Agamotto uses dynamic checkpoints to speed up the analysis. The evaluation was performed using a simple harness that loads and unloads a target driver, which is the only harness supported by Agamotto. Targeted interrupt injection was enabled for each driver. Each driver was analyzed for three hours and no modifications were applied to the driver code. The results of the evaluation can be seen in Table 4. On average VIA improves the number of executed units per second by a factor of 1915 and 2706 for the delayed and non-delayed versions respectively. In
Table 4: Comparison of executions per second and percentage of driver code covered, between Agamotto [56] and VIA without (-D) and with (-ND) delay reduction, averaged over three hours. The evaluation was performed using the basic harness module, which performs one driver load/unload cycle. The coverage is calculated as the percentage of covered trace-pc instrumented instructions divided by the total number of trace-pc instrumented instructions.

\begin{tabular}{lrrrrrrr}
\hline & \multicolumn{3}{c}{ \# Executions / } & & \multicolumn{2}{c}{ \% Code Covered } \\
\cline { 2 - 4 } \cline { 7 - 8 } & Agamotto & VIA-D & VIA-ND & & Agamotto & VIA-ND \\
\hline 8139cp & 1.39 & 1336.00 & 1275.50 & & 4 & 11 \\
e100 & 0.45 & 64.00 & 710.50 & & 3 & 10 \\
e1000 & 0.73 & 1.00 & 66.50 & & 4 & 16 \\
e1000e & 0.74 & 28.00 & 886.00 & & 2 & 9 \\
gve & 1.06 & 1.00 & 732.19 & & 5 & 18 \\
ne2k-pci & 0.35 & 6604.00 & 6332.00 & & 1 & 1 \\
nvme & 0.79 & 0.02 & 1.55 & & 11 & 25 \\
rocker & 0.64 & 2752.00 & 4227.50 & & 1 & 2 \\
sungem & 0.98 & 3144.00 & 6199.50 & & 6 & 8 \\
sunhme & 1.14 & 786.00 & 1250.50 & & 14 & 18 \\
vmxnet3 & 0.74 & 2557.00 & 2735.00 & & 7 & 13 \\
\hline
\end{tabular}

addition, we calculate the percentage of driver code covered by dividing the number of covered trace-pc instrumented instructions by the number of all trace-pc instrumented instructions within the respective driver. On average VIA improves the percentage of covered driver code by a factor of 2.26. During the analysis VIA repeatedly triggered two bugs in the gve and nvme drivers, while Agamotto triggered none.

To evaluate the improvement through delay reduction, we measured the difference in code block coverage and executions per second with and without the optimization. We ran one instance of VIA for each driver listed in Table 2. The execution was terminated after three hours, or after 128 restarts in cases where bugs were triggered shortly after the start of the analysis. To improve the analysis results, we patched identified bugs when possible, as detailed in our repository [1]. For the evaluation of network drivers, we used the more elaborate harness components for network, character and block device drivers, that are provided by VIA. These components load the driver, obtain the resources created by the driver, perform a small set of system calls on the resources and unload the driver again. Each harness contains a concurrent thread that blocks until a workload initiated by the driver enters a blocked state, in which case the routine is woken to trigger an interrupt. All the VirtIO drivers were analyzed in simulation mode.

Table 5 shows the difference in execution speed between the optimized and the un-optimized version. To account for differences in performance due to increased code coverage, we additionally list the total number of covered code blocks. The table shows that reducing delays issued by driver code dramatically increases performance for most drivers without a negative impact on code coverage. We measure an average performance improvement by a factor of 163 
Table 5: Comparison of executions per second and code block coverage as reported by libfuzzer without (-D) and with (-ND) delay reduction, averaged over three hours. The evaluation was performed using the extended harness modules, which interact with resources instantiated by the target drivers.

\begin{tabular}{|c|c|c|c|c|c|}
\hline \multirow[b]{3}{*}{$8139 \mathrm{cp}$} & \multicolumn{3}{|c|}{ \# Executions / s } & \multicolumn{2}{|c|}{ \# Blocks } \\
\hline & \multirow{2}{*}{$\begin{array}{r}\text { VIA-D } \\
1.32\end{array}$} & \multicolumn{2}{|c|}{$\begin{array}{l}\text { VIA-ND } \\
\text { (Increase) }\end{array}$} & \multirow{2}{*}{$\begin{array}{c}\text { VIA-D } \\
1038\end{array}$} & \multirow{2}{*}{$\begin{array}{r}\text { VIA-ND } \\
1040\end{array}$} \\
\hline & & 122.41 & $\times 92.58$ & & \\
\hline acpi & 8.00 & 8.00 & $\times 1.0$ & 71 & 71 \\
\hline e100 & 63.19 & 231.98 & $\times 3.67$ & 573 & 569 \\
\hline e1000 & 3.00 & 259.06 & $\times 86.35$ & 1427 & 1535 \\
\hline e1000e & 0.70 & 111.25 & $\times 158.92$ & 1386 & 1579 \\
\hline gve & 2.00 & 636.22 & $\times 318.11$ & 147 & 594 \\
\hline ne $2 \mathrm{k}-\mathrm{pci}$ & 1408.00 & 1658.00 & $\times 1.18$ & 31 & 31 \\
\hline nvme & 0.02 & 0.88 & $\times 44.0$ & 260 & 291 \\
\hline qemu-fw-cfg & 1254.00 & 1341.0 & $\times 1.06$ & 35 & 37 \\
\hline rocker & 171.01 & 203.25 & $\times 1.19$ & 181 & 184 \\
\hline sungem & 6.01 & 59.04 & $\times 9.82$ & 924 & 1032 \\
\hline sunhme & 195.00 & 428.00 & $\times 2.19$ & 1025 & 1030 \\
\hline tpm-tis & 2.00 & 857.00 & $\times 428.50$ & 150 & 326 \\
\hline vio-balloon & 1291.00 & 1328.00 & $\times 1.03$ & 281 & 281 \\
\hline vio-blk & 625.00 & 624.00 & $\times 1.00$ & 333 & 333 \\
\hline vio-console & 349.00 & 444.00 & $\times 1.27$ & 352 & 352 \\
\hline vio-crypto & 270.00 & 277.00 & $\times 1.03$ & 258 & 258 \\
\hline vio-input & 393.00 & 635.00 & $\times 1.62$ & 299 & 299 \\
\hline vio-net & 553.00 & 400.00 & $\times 0.72$ & 1250 & 1257 \\
\hline vio-rng & 1.00 & 2282.00 & $\times 2282.00$ & 238 & 239 \\
\hline vmxnet3 & 37.07 & 59.94 & $\times 1.62$ & 51 & 51 \\
\hline
\end{tabular}

through reducing delays. However, in some cases, we see either no significant improvement or even a slight reduction in performance. We determined two distinct causes of this behavior. In the first case, we see that the code block coverage for drivers with little or no improvement is low. Therefore, it is likely that the driver initialization failed early before any major interaction with the device was performed. The second case covers VirtIO drivers. These drivers are specifically designed for software device back-ends and do not rely on timeouts as much as device drivers that can also interact with real hardware. We recommend disabling delay optimization in cases where it does not increase overall performance.

We focused on the rocker driver to evaluate whether targeted interrupt injection improves the bug-finding capabilities of VIA. The rocker driver was selected for this evaluation because it requires multiple interrupts to be triggered during its initialization. If no interrupt is triggered within a fixed time window, the initialization fails. To evaluate the effectiveness of the optimization, we ran two instances of VIA: one instance used targeted interrupt injection; the other injected interrupts at random time intervals ranging from $1-1000$ nanoseconds. The experiment was repeated over 1000 runs and each run was started with an empty corpus. Figure 6 compares the average time in seconds required to trigger

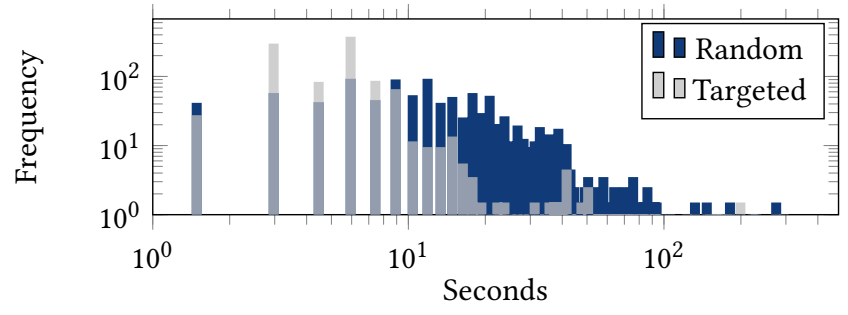

Figure 6: Distribution of the time in seconds required to trigger a bug in the rocker driver with targeted and random interrupt injection.

Table 6: Time required to trigger the first Bug (TTB) in seconds for each examined driver with a known bug, averaged over 128 runs. Patches were applied to avoid low severity bugs due to assertion failures, unbounded allocations and deadlocks.

\begin{tabular}{lr|lr|lr}
\hline & TTB (s) & & TTB (s) & & TTB (s) \\
\hline $8139 \mathrm{cp}$ & 4.60 & acpi & 1.87 & e100 & 1424.80 \\
e1000 & 51.54 & e1000e & 1650.98 & gve & 542.82 \\
nvme & 0.37 & qemu-fw & 2.03 & rocker & 6.78 \\
sungem & 1.50 & tpm-tis & * $7200+$ & vio-balloon & 37.86 \\
vio-blk & 20.24 & vio-console & 18.28 & vio-crypto & 18.92 \\
vio-net & 4.93 & vio-ring & 132.34 & vio-rng & 2.08 \\
vmxnet3 & 1.89 & & & & \\
\hline
\end{tabular}

"Estimate based on few runs, since the bug could not be triggered reliably.

the first bug. Overall, the optimized version took on average of 6.78 seconds to trigger the bug, whereas the unoptimized version took 17.66 seconds. Targeted interrupt injection thereby reduces the time required to trigger the bug by a factor of 2.6 .

To evaluate the overall effectiveness of VIA in uncovering bugs in the $\mathrm{HV}$ interface of device drivers, we measured the elapsed time until the first bug is triggered and averaged the measurement over 128 runs. For this evaluation, we applied the modifications to remove complex checks on data provided by the fuzzer as detailed above. In addition, we applied patches to avoid assertion failures, unbounded allocations and deadlocks. Table 6 shows the results from this evaluation. For most drivers, the first bug is triggered within the first few minutes of fuzzing, with many bugs being triggered in under one minute of analysis. This result demonstrates the effectiveness of VIA in finding such issues, but also underlines the extent of missing sanitization of data provided by the HV in the Linux kernel code-base.

\section{RELATED WORK}

Hardware Interface Analysis. Work on analyzing the hardware side interface of device drivers has mainly focused on the USB interface, with limited support for PCI and other hardware buses. Generic approaches are usually based on symbolic execution or require specific hardware devices. 
Syzkaller-USB [27] adapts the Linux kernel to inject USB packets via the system call interface directly. In order to avoid the requirement for such modifications to the target operating system, a virtual device can be used to forward the fuzzing input. This approach is implemented by USBFuzz [50] and Spenneberg et al. [57] to analyze USB device drivers. Agamotto [56] implements a similar approach using a virtual PCI device. Keil et al. [39] use a virtual device to emulate a specific WiFi chip in order to analyze its respective device driver. Reviewing these approaches, we found their performance to be insufficient. Both Agamotto and USBFuzz cite an average of multiple seconds of execution time per test case. Further, none of the existing techniques can be applied to directly target the DMA interface and lack support for relevant device classes such as VirtIO and Platform.

Symdrive [53], POTUS [49] and DDT [41] facilitate the symbolic execution capabilities provided by S2E [24] to systematically explore driver state. While symbolic execution can analyze hardware interfaces in a generic way, performance is often limited due to path explosion and expensive constraint solving. SADA [20] proposes a static approach to detect several classes of unsafe DMA accesses combined with lightweight symbolic execution. While SADA improves the performance issues of pure symbolic execution methods it incurs disadvantages due to the static nature of the analysis. For example, bugs depending on the runtime state of the driver such as bugs caused by improper handling of failed driver initialization are difficult to detect with purely static approaches. Further, due to incomplete symbolic analysis SADA produces false positives which have to be manually analyzed.

Periscope [55] presents a bus agnostic approach to intercept on device communication between hardware and an operating system and uses this method to implement a fuzzer. To analyze a driver Periscope requires the corresponding device and only analyzes bottom-half interrupt handlers. Driver (de-)initialization, interrupt generation and top-half interrupt handling are excluded from the analysis. Markettos et al.[45] describe a custom hardware component that can be attached to the PCI port in order to trigger bugs in device drivers communicating over that interface. A similar approach based on custom hardware is presented by TTWE [59]. In comparison to these approaches, VIA does not require specific hardware.

Virtualization-Free Kernel Fuzzing. Janus [63] and Hydra [40] utilize the userspace kernel environment provided by LKL to quickly reset state during fuzzing via fork. Based on this primitive, they build a dynamic analysis framework targeted at finding bugs in Linux file system implementations. These approaches, however, target file system implementations and lack support for hardware interface analysis.

Attacks on SEV and TDX. Researchers have already presented security issues in AMD SEV and SEV-ES under the threat model of a malicious HV. The discovered vulnerabilities rely on the missing protection of Second Level Address Translation [30, 47] and missing memory integrity protection from software attacks $[25,43,61]$. Both issues are addressed in the designs of AMD SEV-SNP and Intel TDX. In comparison, the vulnerabilities presented in Section 6.2 are purely software bugs and cannot be protected against through only memory encryption and integrity protection. The discovered bugs do not apply to a particular confidential computing solution, but cover all solutions which rely on devices provided by a malicious HV. Radev and Morbitzer [52] discovered new attack vectors for SEV-ES VMs based on trusting the HV with the emulation of few special instructions, the creation of MMIO regions, and the attachment of VirtIO devices. While the authors suggest that trusting virtualized devices can impact the security of a SEV-ES VM, they only examine the risks for the VM in delegating cryptographic operations to the device virtio-crypto and in using the device virtio-rng as a source of entropy. Our work also confirms the security implications for protected VMs introduced by devices attached to the VM. However, we also provide an automated, scalable approach for discovering software bugs in Linux drivers.

\section{DISCUSSION}

Bug Exploitability. Software bugs in a protected VM carry a high risk since a malicious HV holds fine-grained control over the VM's execution from the initial boot stage [52]. For example, a malicious HV may track the VM's execution to execute an attack at the precise time [52], infer address space information via side-channels [43], or employ multiple malicious virtual devices to combine bugs in various drivers. The exploitability of the discovered bugs and exploitation steps are agnostic to the VM protection technology, but rather depend on the VM's Linux kernel self-protection configuration [38]. For example, all discovered device-shared pointer bugs are exploitable if the virtual offset of the Linux kernel image becomes known to the attacker via another bug or side-channel.

Reducing the trusted computing base can be an effective approach for reducing the amount of software which contains exploitable bugs and requires validations under this new threat model. The early publicly available implementation of Intel TDX attempts to limit the number of buggy device drivers by adding a device filter [35]. The filter blocks discovery of PCI and ACPI devices that are not present in a pre-compiled list. However, the discovered VirtIO / swiotlb bugs from Section 6.2 are applicable to the VirtIO devices featured in the Intel TDX device filter at the time of writing. Additionally, cloud providers may have different device and software stack requirements, which would require adjustments to the list of allowed devices. For example, we determined that the Google Confidential VM does not utilize any of the allowed devices in the TDX device filter.

State Accumulation. VIA avoids state accumulation by re-loading the analyzed driver after each fuzzing iteration. However, some state might still persist and influence future iterations. In cases where state accumulation becomes a problem the fuzzing process can be forked as described by Xu et al. [63] to provide a clean state for each new iteration. VIA supports libfuzzer's Fork Mode [2] to periodically reset the state of the kernel environment.

Limitations. LKL handles concurrency by managing different posix threads that are synchronized via semaphores. If one thread terminates or stalls its execution, it releases the semaphore, and a new thread can be scheduled. Therefore, there is no implicit preemption in LKL, which limits its capabilities in finding race conditions. 
As described in Section 6, VIA requires complex checks such as checksum comparisons to be removed manually. While we do not address such issues directly in this work, we believe that by moving the analysis to userspace, VIA can benefit more readily from recent advances in the field. For example, approaches based on dataflow tracing [54] are currently only available in userspace and can be integrated into VIA.

Disclosure Response. The discovered bugs were reported to several entities including AMD, Intel, Google, driver maintainers and the Linux kernel security, network and virtualization mailing lists. It was recommended to publicize discovered issues, since the threat model of current Linux releases does not yet consider malicious virtual devices. As the bugs do not affect core products of producers and customers of protected virtual machine technologies, they need to be addressed by driver maintainers. Several of the bugs uncovered in swiotlb, virtio_*, vmxnet 3 and gve drivers have been addressed by the respective maintainers.

\section{CONCLUSIONS}

We presented VIA: a framework for dynamic analysis of drivers under the threat model of a malicious HV. VIA was applied to 22 drivers in Linux 5.10.0-rc6, which covered network drivers, VirtIObased drivers, and platform drivers. The evaluation found 50 bugs from a variety of classes and initiated multiple patches to the Linux kernel. We implement Proof-of-Concept exploits for three bug classes that gain code execution or corrupt memory inside an AMD SEV-ES VM. The described exploits demonstrate how the capabilities of a malicious HV, such as control over virtual devices, fine grained control over VM execution as well as information about the VM execution state, can be used to craft reliable exploits. The majority of bugs were reported to the driver maintainers, and the security implications were responsibly disclosed.

Unlike other solutions for driver analysis, VIA carefully considers the capabilities of the HV and the semantics of the DMA API to detect more cases of improper input sanitization and anti-patterns in device programming. VIA provides a coverage-driven in-process userspace fuzzer built upon libfuzzer and LKL. Compared to existing approaches, VIA reduces setup overhead by moving the analysis into a userspace program. In combination with targeted optimizations of the userspace kernel environment, VIA drastically improves dynamic analysis throughput. To the best of our knowledge, VIA presents the first approach to analyze the device driver interface under the new threat model of protected virtual machines.

Our results suggest that many Linux device drivers do not correctly sanitize device-provided data. Under the threat model of AMD SEV-SNP and Intel TDX, this presents a serious security risk for the protected VM, which may be exploited through a software driver bug. Thus, the software operating under such a threat model should reduce the interfaces with untrusted entities and should rely on established methods for testing the remaining interfaces.

\section{ACKNOWLEDGMENTS}

The authors would like to thank our shepherd, Fengwei Zhang, and the anonymous reviewers for their valuable feedback. This material is based upon work partially supported by the European Commission under the Horizon 2020 Programme (H2020) as part of the LOCARD project (G.A. no. 832735). Any opinions, findings, and conclusions or recommendations expressed in this material are those of the authors and do not necessarily reflect the views of our funding agencies.

\section{REFERENCES}

[1] 2021. Github anonymous repository: analysis of discovered bugs in device drivers. https://github.com/fuzzsa/fuzzsa-bugs.

[2] 2021. libFuzzer: a library for coverage-guided fuzz testing. https://lvm.org/docs/ LibFuzzer.html. Accessed: 2021-21-01.

[3] 2021. Patch: gve: Add NULL pointer checks when freeing irqs. https://github. com/torvalds/linux/commit/e96b491a0ffa35a8a9607c193fa4d894ca9fb32f.

[4] 2021. Patch: gve: Update mgmt_msix_idx if num_ntfy changes. https://github. com/torvalds/linux/commit/5218e919c8d06279884aa0baf76778a6817d5b93.

[5] 2021. Patch: swiotlb: Validate bounce size in the sync/unmap path. https://lore. kernel.org/patchwork/patch/1364048/.

[6] 2021. Patch: Untrusted device support for virtio. https://lists.linuxfoundation. org/pipermail/virtualization/2021-June/054685.html.

[7] 2021. Patch: virtio-ring: maintain next in extra state for packed virtqueue. https:// lore.kernel.org/virtualization/20210421032117.5177-2-jasowang@redhat.com/.

[8] 2021. Patch: virtio-ring: store DMA metadata in desc_extra for split virtqueue. https://lore.kernel.org/patchwork/patch/1416241/.

[9] 2021. Patch: virtio_net: Fix error code in probe(). https://lkml.org/lkml/2020/12/ 28/1647.

[10] 2021. Patch: virtio_ring: cut and paste bugs in vring_create_virtqueue_packed(). https://lkml.org/lkml/2020/12/28/687.

[11] 2021. Patch: virtio_ring: Fix two use after free bugs. https://lkml.org/lkml/2020/ $12 / 28 / 828$.

[12] 2021. Patch: vmxnet3: Remove buf_info from device accessible structures. https://git.kernel.org/pub/scm/linux/kernel/git/netdev/net-next.git/ commit/drivers/net/vmxnet3?id=de1da8bcf40564a2adada2d5d5426e 05355f66e 8

[13] 2021. Platform Devices and Drivers. https://www.kernel.org/doc/html/latest/ driver-api/driver-model/platform.html. Accessed: 2021-21-01.

[14] 2021. Virtual I/O Device (VIRTIO) Version 1.1. https://docs.oasis-open.org/virtio/ virtio/v1.1/csprd01/virtio-v1.1-csprd01.html. Accessed: 2021-21-01.

[15] Advanced Micro Devices. 2020. AMD SEV-SNP: Strengthening VM Isolation with Integrity Protection and More.

[16] Al Danial. 2021. Count Lines of Code. https://github.com/AlDanial/cloc. Accessed: 2021-08-06.

[17] AMD. 2021. SEV-SNP OVMF. https://github.com/AMDESE/ovmf.

[18] François Amigorena. 2019. Why SMBs Still do not Trust Cloud Storage Providers to Secure their Data. https://www.infosecurity-magazine.com/opinions/smbtrust-cloud-storage-1-1/. Accessed: 2021-21-01.

[19] Cornelius Aschermann, Sergej Schumilo, Tim Blazytko, Robert Gawlik, and Thorsten Holz. 2019. REDQUEEN: Fuzzing with Input-to-State Correspondence. Ndss (2019). https://doi.org/10.14722/ndss.2019.23xxx

[20] Jia-Ju Bai, Tuo Li, Kangjie Lu, and Shi-Min Hu. 2021. Static Detection of Unsafe DMA Accesses in Device Drivers. In 30th USENIX Security Symposium (USENIX Security 21). USENIX Association. https://www.usenix.org/conference/ usenixsecurity21/presentation/bai

[21] Robert Buhren, Christian Werling, and Jean-Pierre Seifert. 2019. Insecure Until Proven Updated: Analyzing AMD SEV's Remote Attestation. In ACM Conference on Computer and Communications Security (CCS).

[22] Cfir Cohen. 2019. AMD-SEV: Platform DH key recovery via invalid curve attack: (CVE-2019-9836). https://seclists.org/fulldisclosure/2019/Jun/46. Accessed: 201922-05.

[23] Peng Chen, Jianzhong Liu, and Hao Chen. 2019. Matryoshka: fuzzing deeply nested branches. (2019). arXiv:1905.12228

[24] Vitaly Chipounov, Volodymyr Kuznetsov, and George Candea. 2012. The S2E Platform: Design, Implementation, and Applications. ACM Trans. Comput. Syst. 30, 1, Article 2 (Feb. 2012), 49 pages. https://doi.org/10.1145/2110356.2110358

[25] Zhao-Hui Du, Zhiwei Ying, Zhenke Ma, Yufei Mai, Phoebe Wang, Jesse Liu, and Jesse Fang. 2017. Secure Encrypted Virtualization is Unsecure. arXiv:1712.05090 [cs.CR] https://arxiv.org/abs/1712.05090

[26] George Dunlap, Samuel King, Murtaza Basrai, and Peter M Chen. 2002. ReVirt: Enabling Intrusion Analysis through Virtual-Machine Logging and Replay. (2002).

[27] Google. 2019. syzkaller - kernel fuzzer. https://github.com/google/syzkaller.

[28] Google. 2020. Confidential VM and Compute Engine. https://cloud.google.com/ compute/confidential-vm/docs/about-cvm.

[29] PCI Special Interest Group. 1998. PCI Local Bus Specification. Chapter 6.

[30] Felicitas Hetzelt and Robert Buhren. 2017. Security Analysis of Encrypted Virtual Machines. In International Conference on Virtual Execution Environments. 
[31] Guerney Hunt, Richard Boivie, Eric Hall, Elaine Palmer, Dimitrios Pendarakis, and Enriquillo Valdez. 2018. Supporting protected computing on IBM Power Architecture. https://developer.ibm.com/articles/l-support-protected-computing/ Accessed: 2021-21-01.

[32] Intel. 2020. Architecture Specification: Intel Trust Domain Extensions (Intel TDX) Module. https://software.intel.com/content/dam/develop/external/us/en/ documents/intel-tdx-module-1eas.pdf Accessed: 2021-15-01.

[33] Intel. 2020. Intel Trust Domain Extensions (whitepaper).

[34] Intel. 2020. Trust Domain Extensions. https://github.com/intel/tdx.

[35] Intel. 2020. x86/tdx: Add device filter support for x86 TDX platform. https //github.com/intel/tdx/commit/6789eee52aab8985e49b362379fab73aa3eecde2.

[36] S. Jin, J. Ahn, S. Cha, and J. Huh. 2011. Architectural support for secure virtualization under a vulnerable hypervisor. In 2011 44th Annual IEEE/ACM International Symposium on Microarchitecture (MICRO). 272-283.

[37] David Kaplan, Jeremy Powell, and Tom Woller. 2016. White Paper AMD Memory Encryption. http://amd-dev.wpengine.netdna-cdn.com/wordpress/media/2013/ 12/AMD_Memory_Encryption_Whitepaper_v7-Public.pdf.

[38] Kees Cook. 2017. Linux Kernel Self-Protection. https://www.usenix.org/system/ files/login/articles/login_spring17_04_cook.pdf.

[39] Sylvester Keil and Clemens Kolbitsch. 2007. Stateful Fuzzing of Wireless Device Drivers in an Emulated Environment.

[40] Seulbae Kim, Meng Xu, Sanidhya Kashyap, Jungyeon Yoon, Wen Xu, and Taesoo Kim. 2019. Finding semantic bugs in file systems with an extensible fuzzing framework. Proceedings of the 27th ACM Symposium on Operating Systems Principles (2019).

[41] Volodymyr Kuznetsov, Vitaly Chipounov, and George Candea. 2010. Testing Closed-Source Binary Device Drivers with DDT. In Proceedings of the 2010 USENIX Conference on USENIX Annual Technical Conference (Boston, MA) (USENIXATC'10) USENIX Association, USA, 12.

[42] Mengyuan Li, Yinqian Zhang, and Zhiqiang Lin. 2020. CROSSLINE: Breaking "Security-by-Crash" based Memory Isolation in AMD SEV.

[43] Mengyuan Li, Yinqian Zhang, Zhiqiang Lin, and Yan Solihin. 2019. Exploiting Unprotected I/O Operations in AMD's Secure Encrypted Virtualization. In USENIX Security Symposium.

[44] LLVM. 2021. Address Sanitizer. https://clang.llvm.org/docs/AddressSanitizer. html. Accessed: 2021-08-06.

[45] A. Theodore Markettos, Colin Rothwell, Brett F. Gutstein, Allison Pearce, Peter G. Neumann, Simon W. Moore, and Robert N. M. Watson. 2019. Thunderclap: Exploring Vulnerabilities in Operating System IOMMU Protection via DMA from Untrustworthy Peripherals. February (2019). https://doi.org/10.14722/ndss.2019. 23194

[46] Mathias Morbitzer, Manuel Huber, and Julian Horsch. 2019. Extracting Secrets from Encrypted Virtual Machines. In ACM Conference on Data and Application Security and Privacy (CODASPY).

[47] Mathias Morbitzer, Manuel Huber, Julian Horsch, and Sascha Wessel. 2018. SEVered: Subverting AMD's Virtual Machine Encryption. In European Workshop on Systems Security (EuroSEC).

[48] Karsten Nohl and Jakob Lell. 2014. BadUSB-On Accessories That Turn Evil. Black Hat USA 1, 9 (2014), 1-22.

[49] James Patrick-Evans, Lorenzo Cavallaro, and Johannes Kinder. 2017. POTUS: Probing Off-The-Shelf USB Drivers with Symbolic Fault Injection. In 11th USENIX Workshop on Offensive Technologies (WOOT 17). USENIX Association, Vancouver, BC. https://www.usenix.org/conference/woot17/workshopprogram/presentation/patrick-evans

[50] Hui Peng and Mathias Payer. 2020. USBFuzz: A framework for fuzzing USB drivers by device emulation. Proceedings of the 29th USENIX Security Symposium (2020), 2559-2575.

[51] Octavian Purdila, Lucian Adrian Grijincu, and Nicolae Tapus. 2010. LKL: The Linux kernel library. Proceedings - 9th RoEduNet IEEE International Conference, RoEduNet 2010 (2010), 328-333.

[52] Martin Radev and Mathias Morbitzer. 2020. Exploiting Interfaces of Secure Encrypted Virtual Machines. arXiv preprint arXiv:2010.07094 (2020).

[53] Matthew J. Renzelmann, Asim Kadav, and Michael M. Swift. 2012. SymDrive Testing Drivers without Devices. In Proceedings of the 10th USENIX Conference on Operating Systems Design and Implementation (Hollywood, CA, USA) (OSDI'12). USENIX Association, USA, 279-292.

[54] Gabriel Ryan, Abhishek Shah, Dongdong She, Koustubha Bhat, and Suman Jana. 2019. Fine Grained Dataflow Tracking with Proximal Gradients. (2019) arXiv:1909.03461

[55] Dokyung Song, Felicitas Hetzelt, Dipanjan Das, Chad Spensky, Yeoul Na, Stijn Volckaert, Giovanni Vigna, Christopher Kruegel, Jean-Pierre Seifert, and Michael Franz. 2019. PeriScope: An Effective Probing and Fuzzing Framework for the Hardware-OS Boundary. February (2019). https://doi.org/10.14722/ndss.2019. 23176

[56] Dokyung Song, Felicitas Hetzelt, Jonghwan Kim, Brent ByungHoon Kang, JeanPierre Seifert, and Michael Franz. 2020. Agamotto: Accelerating Kernel Driver Fuzzing with Lightweight Virtual Machine Checkpoints. In 29th USENIX Security Symposium (USENIX Security 20). USENIX Association, 2541-2557. https://www. usenix.org/conference/usenixsecurity20/presentation/song

[57] Ralf Spenneberg. 2014. Don ' $t$ trust your USB ! How to find bugs in USB device drivers.

[58] Jakub Szefer and Ruby B. Lee. 2012. Architectural Support for Hypervisor-secure Virtualization. SIGPLAN Not. 47, 4 (March 2012), 437-450. https://doi.org/10. $1145 / 2248487.2151022$

[59] Rijnard van Tonder and Herman Engelbrecht. 2014. Lowering the USB Fuzzing Barrier by Transparent Two-Way Emulation. In 8th USENIX Workshop on Offensive Technologies (WOOT 14). USENIX Association, San Diego, CA. https://www. usenix.org/conference/woot14/workshop-program/presentation/van-tonder

[60] Jan Werner, Joshua Mason, Manos Antonakakis, Michalis Polychronakis, an Fabian Monrose. 2019. The SEVerESt Of Them All: Inference Attacks Against Secure Virtual Enclaves. In ACM Symposium on Information, Computer and Communications Security (ASIACCS).

[61] Luca Wilke, Jan Wichelmann, Mathias Morbitzer, and Thomas Eisenbarth. 2020. SEVurity: No Security Without Integrity - Breaking Integrity-Free Memory Encryption with Minimal Assumptions. In IEEE Symposium on Security and Privacy $(S \& P)$.

[62] Y. Xia, Y. Liu, and H. Chen. 2013. Architecture support for guest-transparent VM protection from untrusted hypervisor and physical attacks. In 2013 IEEE 19th International Symposium on High Performance Computer Architecture (HPCA). 246-257. https://doi.org/10.1109/HPCA.2013.6522323

[63] Wen Xu, Hyungon Moon, Sanidhya Kashyap, Po-Ning Tseng, and Taesoo Kim. 2019. Fuzzing File Systems via Two-Dimensional Input Space Exploration. 2019 IEEE Symposium on Security and Privacy (SP) (2019), 818-834.

[64] Fengzhe Zhang, Jin Chen, Haibo Chen, and Binyu Zang. 2011. CloudVisor: Retrofitting protection of virtual machines in multi-tenant cloud with nested virtualization. SOSP'11 - Proceedings of the 23rd ACM Symposium on Operating Systems Principles, 203-216. https://doi.org/10.1145/2043556.2043576 